\title{
Morphological and Physical Properties of Randomly Coupled Multiblock Copolymers: Bisphenol-A Polycarbonate-Poly(oxyethylene) Systems
}

\author{
Toshitake SUZUKI* and Tadao KoTAKA** \\ Department of Macromolecular Science, Faculty of Science, \\ Osaka University, Toyonaka, Osaka 560, Japan
}

(Received June 17, 1982)

\begin{abstract}
For randomly coupled (AB) ${ }_{n}$ type multiblock copolymers of bisphenol-A polycarbonate (PC) and poly(oxyethylene) (POE) with varying $\mathrm{PC} / \mathrm{POE}$ composition and block length, crystallization, melting, and microphase separation were examined by DSC, dynamic mechanical and stress-strain measurements, dielectric relaxation measurements, and polarizing optical microscopy. These processes were strongly dependent on PC/POE composition and block length. The microphase separation became more significant with increasing block length, and various phases (such as PC and POE crystalline and amorphous phases) appeared depending on PC/POE composition. In the copolymers with high POE content $(~ \gtrsim 70 \%)$, a spherulitic structure was found. The spherulites appeared to consist of POE crystallites, POE amorphous segments and PC stacking regions. The PC stacking regions imparted to the specimen toughness against deformation and behaved as crosslinks after the spherulites underwent destruction by stretching of the specimens.
\end{abstract}

KEY WORDS Multiblock Copolymer / Bisphenol-A PolycarbonatePoly(oxyethylene) Copolymer / Microphase Separation / Dynamic Mechanical Property / Stress-Strain Curve / Dielectric Relaxation / Spherulitic Structure /

Recently multicomponent polymers or polymeric alloys have drawn attention owing to their unique physical properties originating from microphase separation. ${ }^{1,2}$ Alternating (AB) ${ }_{n}$-type multiblock copolymers such as segmented polyurethane elastomers $^{3-5}$ may also be included in this class of material when the blocks are sufficiently long to induce microphase separation. We studied the physical properties of $(\mathrm{AB})_{n}$-type multiblock copolymers consisting of bisphenol-A polycarbonate (PC) and poly(oxyethylene) (POE) blocks. ${ }^{6-8} \mathrm{POE}$ is highly crystallizable and has rubbery or soft segments. PC is less easily crystallizable and has glassy or hard segments. We found that the properties of the copolymers changed markedly with varying PC/POE composition and block length. ${ }^{6-8}$ Our results may be summarized as follows. i) Crystallization of POE blocks is hampered by adjoining hard PC blocks, while that of PC blocks is facilitated by soft POE blocks.

ii) The physical properties of multiblock copolymers vary from those of homogeneous random copolymers to those of heterophase block copolymers with increasing block length.

However, our previous work was limited to samples of a rather narrow range of composition and block length. The block copolymers studied were relatively rich in PC (PC/POE ratio was varied from $50 / 50$ to $90 / 10$ ), and the degree of polymerization of PC and POE blocks varied from 2 to 20 and 2 to 68 , respectively. Therefore, it was of interest to study the properties of multiblock copolymers of a much wider range of $\mathrm{PC} / \mathrm{POE}$ composition and block

* Present address: Kanzaki Paper Mfg. Co., Ltd., Amagasaki, Hyogo 660, Japan.

** To whom correspondence should be addressed. 
length. We prepared six well-defined samples, in which the PC/POE ratio was 75/25, 50/50, and 30/ 70 by weight, and two samples of the same composition but having different PC and POE block lengths were synthesized. We carried out differential scanning calorimetry (DSC), dielectric, dynamic mechanical and tensile stress-strain measurements and polarizing microscopic observation. The results are reported in this paper.

\section{EXPERIMENTAL}

Materials. Commercially available polyoxyethylene glycols and bisphenol-A samples were purified as follows: Bisphenol-A was recrystallized from a benzene/methanol (volume ratio 100:5) mixture. Polyoxyethylene glycols with nominal molecular weights $M=3000,2000,1000$, and 600 were fractionally precipitated into $n$-hexane from benzene solutions. These purified polyethers were examined on a gel permeation chromatograph (GPC: Model HLC-801A, Toyo Soda Mfg. Co.) and found to have narrow molecular weight distributions. Reagent-grade pyridine was dried by calcium hydride and further purified by distillation.

Block copolymerization was carried out according to the procedure reported by Goldberg. ${ }^{9}$ Prescribed amounts of bisphenol-A and POE glycol were mixed in pyridine, and phosgene was introduced into the mixture with vigorous stirring over a temperature range from 25 to $30^{\circ} \mathrm{C}$. The product was recovered by adding excess 2 -propanol, and washed with 2-propanol until free of pyridine. The product was then purified by precipitating it into $n$-hexane or methanol from a tetrahydrofuran solution. Powder-form precipitates were dried in a vacuum at $60^{\circ} \mathrm{C}$.

The composition of each product was determined by elemental analysis and UV absorption. The weight average molecular weight was estimated on a Toyo Soda GPC equipped with a refractometer and a low-angle laser light-scattering monitor (LALLS: Model LS-8, Toyo Soda Mfg., Co.). The carrier solvent was chloroform. The characteristics of the copolymer samples are summarized in Table $\mathrm{I}$, in which the samples are coded as BXEY, with $X$ and $Y$ denoting, respectively, the average degree of polymerization of the PC block and that of the POE precursor. The average number $Z$ of $P O E$ oligomers coupled to form a POE block and the value of $X$ were calculated from the monomer feed and product composition assuming random coupling of bisphenol-A units and POE oligomers. For the copolymers, the $\mathrm{PC} / \mathrm{POE}$ ratio was chosen to be roughly $75 / 25,50 / 50$, and $30 / 70$ by weight, and PC and POE block lengths were selected as $X=9$ and 22, $Y=14$ and 46 (75/25 ratio), $X=5$ and $13, Y=23$ and 68 (50/50 ratio) and $X=2$ and 5, $Y=23$ and 68 (30/70 ratio).

Methods. Films were prepared under controlled evaporation of the solvent from a $5 \mathrm{wt} \%$ chloroform solution at room temperature, dried in the air for 3 to 4 days and then under a vacuum of $10^{-2}$ torr for

Table I. Characteristics of copolymer samples ${ }^{\mathrm{a}}$

\begin{tabular}{|c|c|c|c|c|c|c|}
\hline \multirow{2}{*}{$\begin{array}{l}\text { Sample } \\
\text { code } \\
\text { (BXEY) }\end{array}$} & $\begin{array}{l}\text { Bisphenol-A } \\
\text { content in } \\
\text { monomer feed }\end{array}$ & $\begin{array}{c}\text { Bisphenol-A } \\
\text { carbonate } \\
\text { content }\end{array}$ & \multirow[t]{2}{*}{$X^{\mathbf{b}}$} & \multirow[t]{2}{*}{$Y$} & \multirow[t]{2}{*}{$Z^{\mathrm{b}}$} & \multirow[t]{2}{*}{$10^{-4} M_{\mathrm{app}}{ }^{\mathrm{c}}$} \\
\hline & $w t \%$ & $\mathrm{wt} \%$ & & & & \\
\hline B22E46 & 75.0 & 73.1 & 22.9 & 46 & 1.1 & 9.9 \\
\hline B9E14 & 75.1 & 77.2 & 9.6 & 14 & 1.1 & 10.1 \\
\hline B13E68 & 53.5 & 51.8 & 13.9 & 68 & 1.1 & 6.9 \\
\hline B5E23 & 52.7 & 52.3 & 5.5 & 23 & 1.2 & 14.3 \\
\hline B5E68 & 31.1 & 30.0 & 6.1 & 68 & 1.2 & 10.7 \\
\hline B2E23 & 31.1 & 30.0 & 2.8 & 23 & 1.6 & 10.9 \\
\hline
\end{tabular}


2 more days. Differential scanning calorimetry was carried out on a DSC (Rigaku Denki Co., Model 8055 ), in most cases, over a range from 170 to $573 \mathrm{~K}$ at a heating rate of $10 \mathrm{~K} \mathrm{~min}^{-1}$. Dielectric measurements were made in the range from $100 \mathrm{~Hz}$ to $100 \mathrm{kHz}$ on a transformer bridge (General Radio Model 1615A) and an automatic capacitance bridge (Yokogawa Hewlett Packard Model YHP 4070A). Films about $0.3 \mathrm{~mm}$ thick were cut into disks $30 \mathrm{~mm}$ in diameter and coated with evaporated gold electrodes for dielectric measurements. The specimen was mounted on a three-electrode cell in a brass sealing container. ${ }^{10,11}$ Before measurement, the specimen was dried in situ at about $10^{-2}$ torr for $12 \mathrm{hr}$, and the air in the container was replaced by helium. Dynamic mechanical measurements were usually made on a Rheovibron DDV-II (Toyo Baldwin Co.), at $110 \mathrm{~Hz}$ over a temperature range from 150 to $450 \mathrm{~K}$. Tensile stress-strain measurements were made with a tensile tester (Iwamoto Seisakusho, Kyoto) at a strain rate of $400 \% \mathrm{~min}^{-1}$ at room temperature. Spherulitic structures in as-cast films were observed under a polarizing microscope (Nihon Kogaku Mfg., Co.).

\section{RESULTS}

Differential Scanning Calorimetry. Figure 1 shows DSC thermograms designated as the 1st run and 2nd run. The 1st run thermogram refers to an ascast film, and the 2nd to the specimen quenched from $520 \mathrm{~K}$ following the 1 st run measurment. An endothermic peak due to melting of PC crystallites can be seen in a range from 470 to $510 \mathrm{~K}$ for each of the B22E46, B9E14, and B13E68 samples. When quenched from $520 \mathrm{~K}$, which is slightly above the melting temperature $T_{\mathrm{m}}(=490-505 \mathrm{~K})$ of PC crystallites (2nd run), only B13E68 exhibited the PC melting peak preceded by an exothermic peak at $404 \mathrm{~K}$. This exothermic peak was apparently due to the recrystallization of PC segments during the reheating process.

The features of melting and crystallization of POE crystallites were more complicated than those of PC crystallites. All as-cast specimens of B13E68, B2E23, and B5E68 (1st run) exhibited endothermic peaks due to the melting of POE crystallites from 300 to $320 \mathrm{~K}$, while only B2E23 exhibited an exothermic peak due to the crystallization of POE

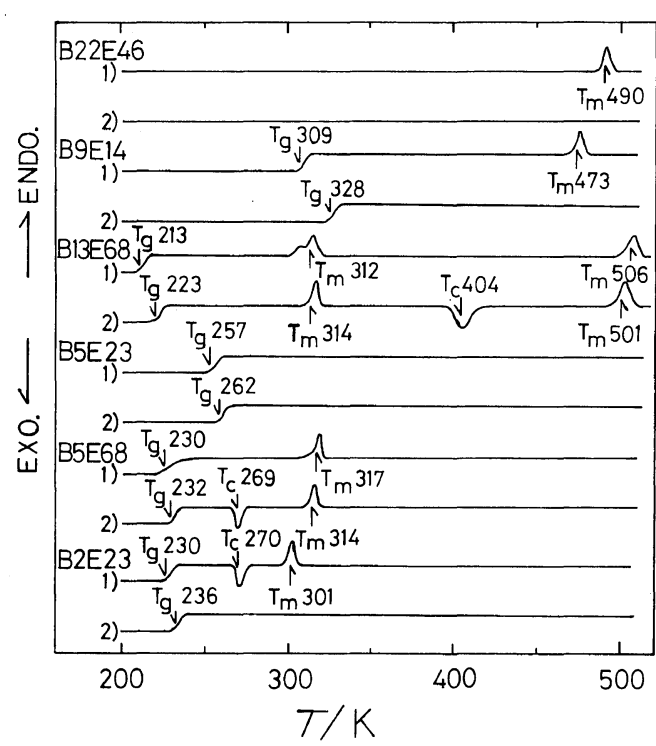

Figure 1. DSC thermograms of the copolymer samples.

segments at about $270 \mathrm{~K}$. On the other hand, when quenched from $520 \mathrm{~K}$ (2nd run), only B13E68 and B5E68 exhibited the POE melting peak, and only B5E68 of these two exhibited the POE recrystallization peak at about $269 \mathrm{~K}$, while B2E23 exhibited only the glass transition at about $236 \mathrm{~K}$.

The glass transition was detected for all samples except B22E46. The glass transition temperature $T_{\mathrm{g}}$ of B9E14 and B5E23 having fairly short blocks were relatively high, being about $20 \mathrm{~K}$ lower than those for the corresponding homogeneous random copolymer estimated by Fox's equations. ${ }^{12}$ Three other samples having long POE blocks had $T_{\mathrm{g}}$ close to those of POE homopolymers (about $220 \mathrm{~K}) .{ }^{13}$ Another interesting feature was that the quenched specimens (2nd run) always had somewhat higher $T_{\mathrm{g}}$ than those of the corresponding as-cast specimens (1st run). Table II summarizes the results from the DSC measurements.

Dynamic Mechanical Properties. Figures 2, 3, and 4 show the temperature dependence of storage Young's modulus $E^{\prime}$ and mechanical loss tangent $\delta$ for the samples containing about $75 \%$ PC (B22E46 and B9E14), those containing 50\% PC (B13E68 and B5E23), and those containing 30\% PC (B5E68 and B2E23), respectively. The short-block polymers of the first two series, B9E14 and B5E23, exhibit a 
Table II. Summary of DSC data of the copolymer samples ${ }^{\mathrm{a}}$

\begin{tabular}{|c|c|c|c|c|c|c|c|c|c|c|}
\hline \multirow{2}{*}{$\begin{array}{c}\text { Code } \\
\text { (BXEY) }\end{array}$} & \multicolumn{5}{|c|}{ As cast specimens } & \multicolumn{5}{|c|}{ Quenched specimens } \\
\hline & $T_{\mathrm{g}}$ & $T_{\mathrm{m}}^{\mathrm{POE}}$ & $\left(X_{\mathrm{c}} / \%\right)$ & $T_{\mathrm{m}}^{\mathrm{PC}}$ & $\left(X_{\mathrm{c}} / \%\right)$ & $T_{\mathrm{g}}$ & $T_{\mathrm{m}}^{\text {POE }}$ & $\left(X_{\mathrm{c}} / \%\right)$ & $T_{\mathrm{m}}^{\mathrm{PC}}$ & $\left(X_{\mathrm{c}} / \%\right)$ \\
\hline B22E46 & - & - & & 490 & (15) & - & - & & - & \\
\hline B9E14 & 309 & - & & 473 & (15) & 328 & - & & - & \\
\hline B13E68 & 213 & 312 & (22) & 506 & (46) & 223 & 314 & (28) & 501 & (55) \\
\hline B5E23 & 257 & - & & - & & 262 & - & & - & \\
\hline B5E68 & 230 & 317 & (48) & - & & 232 & 314 & (38) & - & \\
\hline B2E23 & 230 & 301 & (16) & - & & 236 & - & & - & \\
\hline
\end{tabular}

${ }^{\text {a }}$ Melting $\left(T_{\mathrm{m}}\right)$ and glass transition $\left(T_{\mathrm{g}}\right)$ temperatures for the homopolymers are as follows: $T_{\mathrm{m}}{ }^{\mathrm{PC}}=522 \mathrm{~K}, T_{\mathrm{g}}{ }^{\mathrm{PC}}=$ $418 \mathrm{~K}, T_{\mathrm{m}}{ }^{\mathrm{POE}}=303 \mathrm{~K}$, and $T_{\mathrm{g}}^{\mathrm{POE}}=220 \mathrm{~K}^{7} X_{\mathrm{c}}$ is the degree of crystallinity in $\mathrm{wt} \%$, and was determined by the following equation: $h=C X_{\mathrm{C}} H / 100$, where $h$ is the heat of fusion estimated from DSC thermogram, $H$ the heat of fusion of PC or POE homopolymers $\left(110 \mathrm{~J} \mathrm{~g}^{-1}\right.$ for PC and $188 \mathrm{~J} \mathrm{~g}^{-1}$ for POE), and $C$ the content of PC or POE by weight.

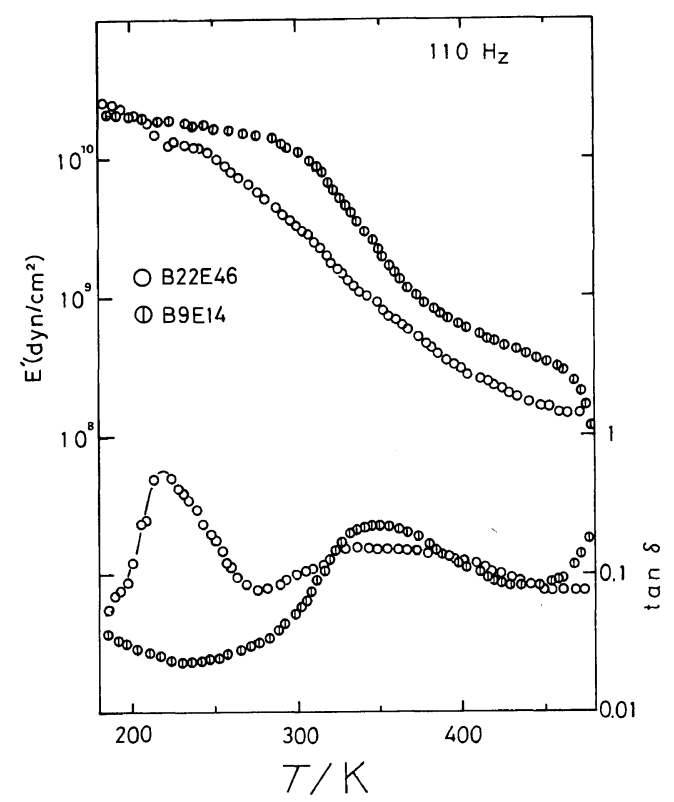

Figure 2. Temperature dependence of storage Young's modulus $E^{\prime}$ and mechanical loss tangent $\delta$ at $110 \mathrm{~Hz}$ for B22E46 and B9E14 samples.

single transition region around 350 and $290 \mathrm{~K}$, respectively, corresponding to the glass transition observed by DSC. On the other hand, the longblock samples, B22E46 and B13E68, exhibit a broad transition region where $E^{\prime}$ decreases gradually and $\tan \delta$ shows a broad peak or a plateau region. For the third group B5E68 and B2E23 samples with large POE content, the temperature dependence of $E^{\prime}$ is strongly influenced by melting

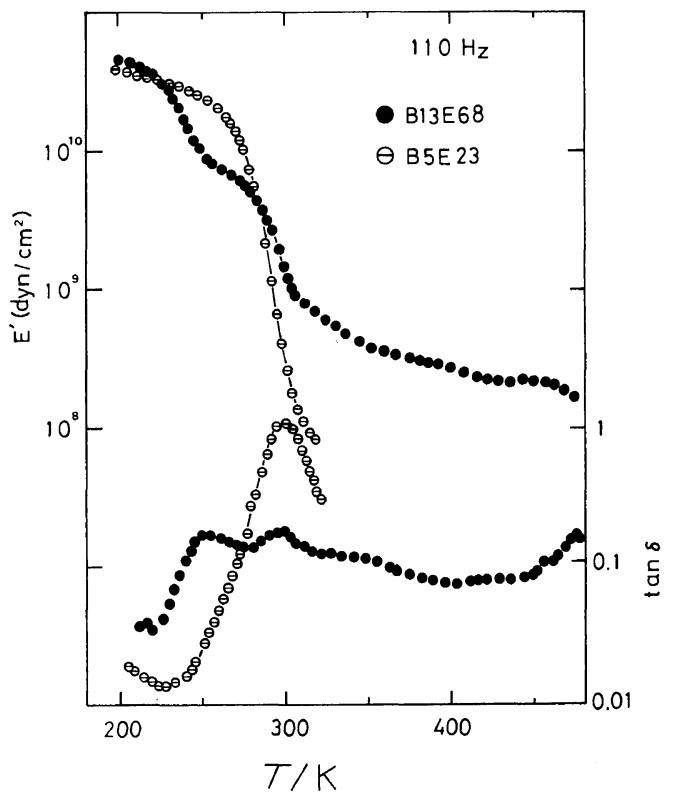

Figure 3. Temperature dependence of $E^{\prime}$ and $\tan \delta$ at $110 \mathrm{~Hz}$ for B13E68 and B5E23 samples.

and crystallization of POE segments. In particular the $E^{\prime}$ versus temperature curve of B2E23 showed anomalous behavior at about 270 and $300 \mathrm{~K}$. That is, $E^{\prime}$ decreased sharply in correspondence to the glass transition observed by DSC, increased steeply at about $270 \mathrm{~K}$ and decreased sharply again at about $300 \mathrm{~K}$. This anomaly appears to correspond to the initiation of crystallization and melting of the POE domains, as observed in the DSC thermogram. Interestingly, when the sample was cooled from a 


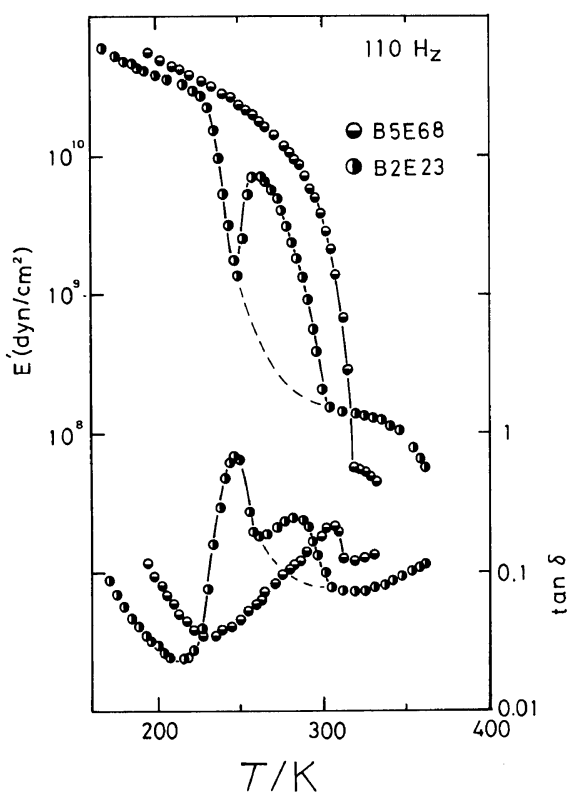

Figure 4. Temperature dependence of $E^{\prime}$ and $\tan \delta$ at $110 \mathrm{~Hz}$ for B5E68 and B2E23 samples.

temperature above $T_{\mathrm{m}}(\simeq 301 \mathrm{~K})$, the anomaly disappeared and the $E^{\prime}$ curve followed the dashed line as shown in Figure 4.

Dielectric Properties. Figures 5, 6, and 7 show the temperature dependence of dielectric constant $\varepsilon^{\prime}$ and loss $\varepsilon^{\prime \prime}$ measured at $1 \mathrm{kHz}$ for the three groups of copolymer samples. The loss curves show two peaks designated as $\alpha$ and $\beta$ from the high temperature side., ${ }^{74,15}$ The $\alpha$-peak temperature $T_{\alpha}$ corresponds to $T_{\mathrm{g}}$ determined by DSC. The $\beta$ peak temperature $T_{\beta}$ does not depend on either block lengths or PC-POE composition, but remains nearly the same as those of POE homopolymers. ${ }^{16}$

Because of ionic conduction, the $\alpha$-peak could not be detected at low frequency. Therefore, $\log$ (frequency $f$ ) versus $T_{\alpha}^{-1}$ plots could be made only for B22E46, B9E14, and B13E68. These plots were described by the Williams-Landel-Ferry (WLF) equation. ${ }^{17}$ From their slopes at $1 \mathrm{kHz}$, the apparent activation energy $E_{\alpha}$ was found to be 330 , 345 , and $420 \mathrm{~kJ} \mathrm{~mol}^{-1}$ for B13E68, B22E46, and B9E14, respectively. The value for B13E68 is comparable to those of the POE homopolymers. ${ }^{16}$ The corresponding plots for the $\beta$-relaxation followed the Arrhenius equation. The value of the activation

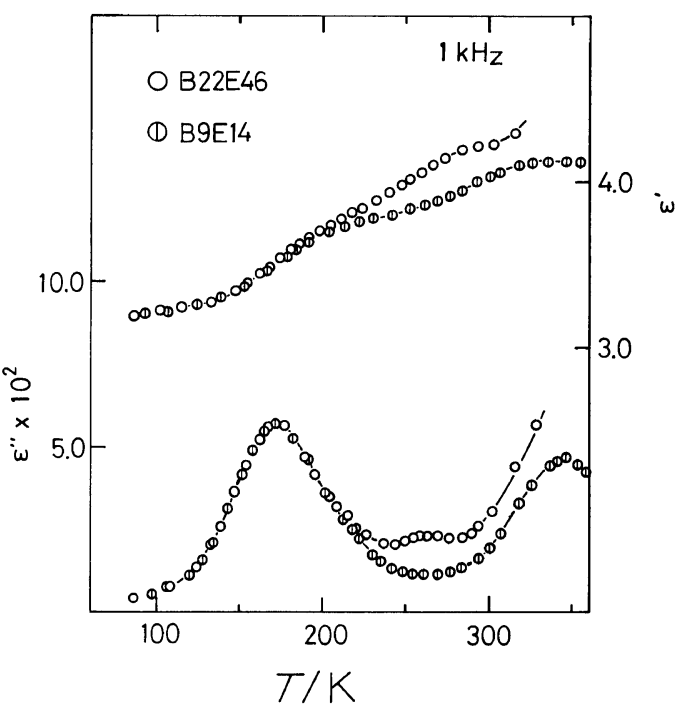

Figure 5. Temperature dependence of dielectric constant $\varepsilon^{\prime}$ and loss $\varepsilon^{\prime \prime}$ at $1 \mathrm{kHz}$ for B22E46 and B9E14 samples.

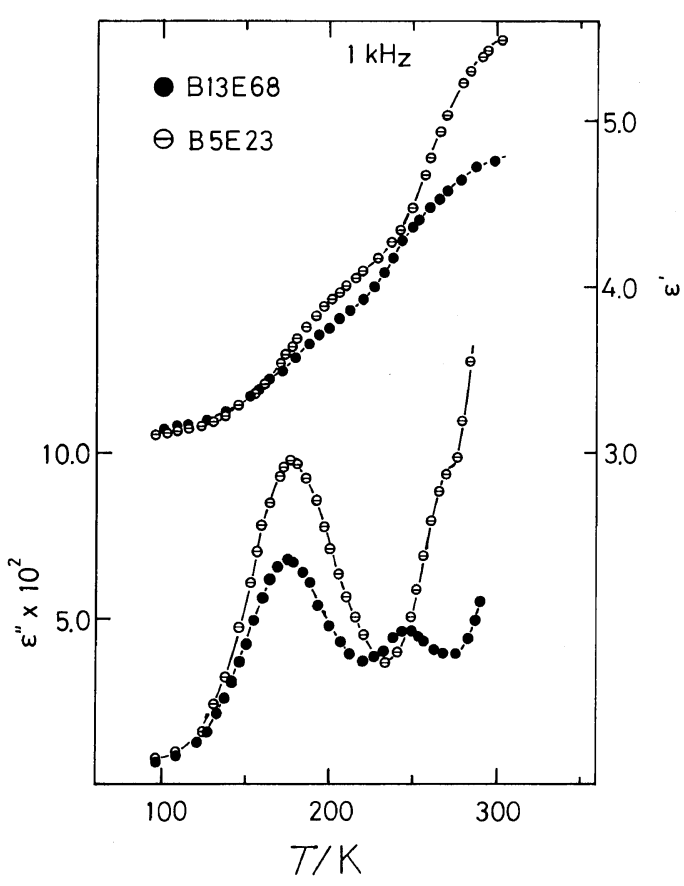

Figure 6. Temperature dependence of $\varepsilon^{\prime}$ and $\varepsilon^{\prime \prime}$ at $1 \mathrm{kHz}$ for B13E68 and B5E23 samples.

energy $E_{\beta}$ was about $36 \mathrm{~kJ} \mathrm{~mol}^{-1}$ for all samples, and was comparable to those of the POE homopolymers. ${ }^{16}$ 


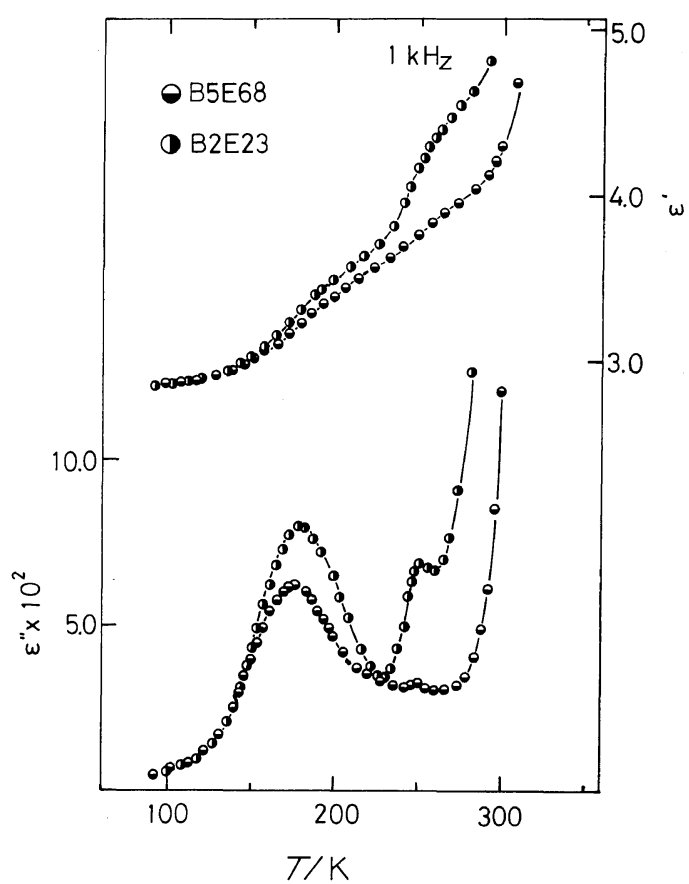

Figure 7. Temperature dependence of $\varepsilon^{\prime}$ and $\varepsilon^{\prime \prime}$ at $1 \mathrm{kHz}$ for B5E68 and B2E23 samples.

In our previous paper, ${ }^{7}$ we concluded that the $\beta$ relaxation might occur as a result of the local inchain motion of POE segments involving only three or four consecutive bonds. The peak hight of this relaxation was roughly in proportion to the $\mathrm{POE}$ amorphous content as calculated from DSC data. Therefore, the $\beta$-peak intensity was the largest for the B5E23 having the largest POE amorphous content. These results support our previous conclusion on the mechanism of the $\beta$-relaxation.

Stress-Strain Behavior. Figure 8 shows stressstrain behavior, and that the tensile property changes from that of a glassy polymer to that of an elastomer with increasing POE content. The tensile property of the samples of high $\mathrm{PC}$ content (B22E46 and B9E14) resembles that of homo-PC, and the tensile recovery is very small. The samples of high POE content (B5E68 and B2E23) are elastomeric and the ultimate elongation is large. However, POE homopolymers show no such behavior because of their high crystallinity.

Figure 8 also shows that the block length is an important factor for tensile strength, especially in

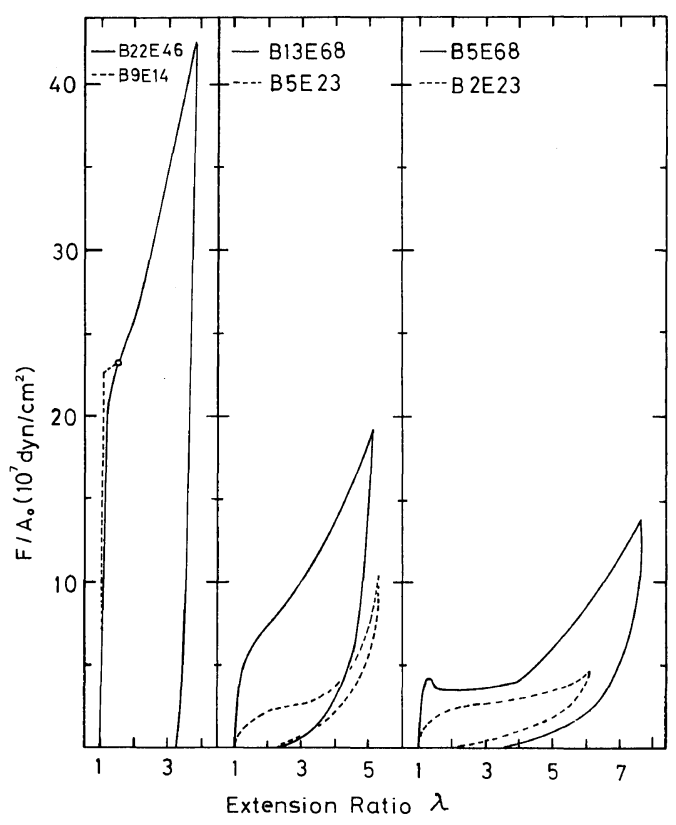

Figure 8. Stress-strain curves for the copolymer samples.

samples of realtively large POE content. Comparing the data of B13E68 and B5E23 or those of B5E68 and B2E23, it is noted that the copolymer becomes increasingly rigid and tough, probably due to progress in microphase separation with increasing block lengths. For B5E68 we observe, by a polarizing microscope and by DSC, yielding and necking due to the destruction of the spherulitic structure. That is, after yielding the spherulitic structure could no longer be observed and the POE-melting peak significantly diminished.

Observation by Polarizing Microscope. Figure 9 shows polarizing optical micrographs of B5E68 and B2E23 taken with white light. Patterns typical of spherulitic structure are noted. The spherulites in B5E68 are relatively large and occupy the entire visual field, while those in B2E23 are small and isolated. From these photographs, it may be expected that the molecular axis orients in either a radial or tangential direction. In order to determine the orientation, interference colors were also examined by a polarizing microscope with white light. The interference color was observed when a sensitive color plate (retardation $=530 \mathrm{~m} \mu$ ) was inserted between the sample and the analyzer 


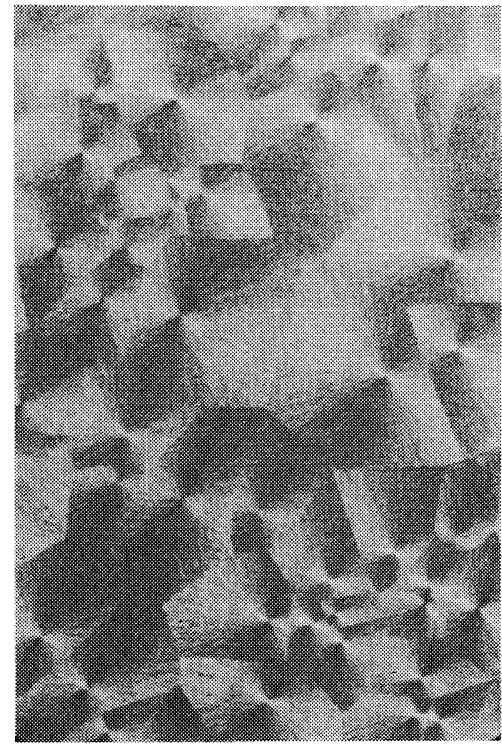

(A)

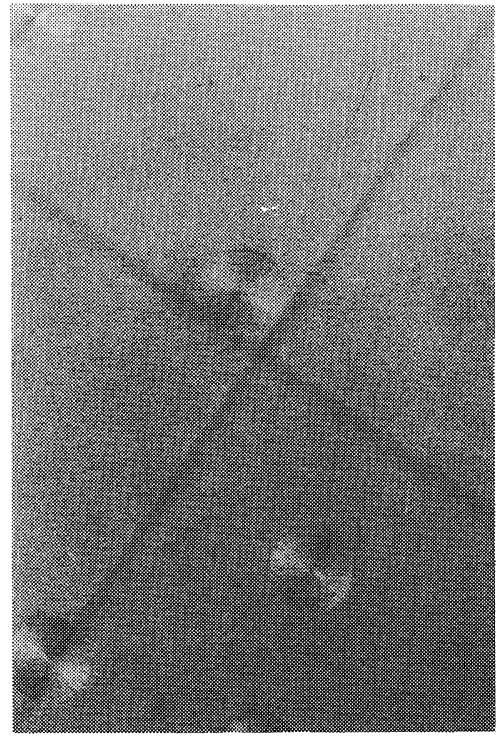

(B)

\section{$0.5 \mathrm{~mm}$}

Figure 9. Polarizing optical micrographs for B5E68 (A) and B2E23 (B) films.

at an angle of $45^{\circ}$ relative to the analyzer. The spherulites exhibited four quadrants colored in blue or yellow under this condition. It was found both for spherulites of B5E68 and B2E23 that the quadrants in the direction of the axis of the sensitive color plate having the largest refractive index were colored yellow, while those at right angles to the axis were colored blue. This indicates the tangential orientation of the molecular axis.

Effects of Thermal Treatments. As can be seen from DSC thermograms, thermal history influences sample morphologies and hence properties. Hence, we compared the properties of annealed and as-cast specimens of the same sample. Figure 10 compares the temperature dependence of $E^{\prime}$ and $\tan \delta$ of ascast and annealed specimens of B5E23. The latter specimen was annealed at $60^{\circ} \mathrm{C}$ for 2 days. The as-cast specimen shows a steep decrease in $E^{\prime}$, suggesting a single-phase structure, and the annealed specimen shows $E^{\prime}$ which begins to decrease at a temperature lower than that of the annealed specimen and has a plateau region in the range from 300 to $400 \mathrm{~K}$. This behavior is characteristic of multi-

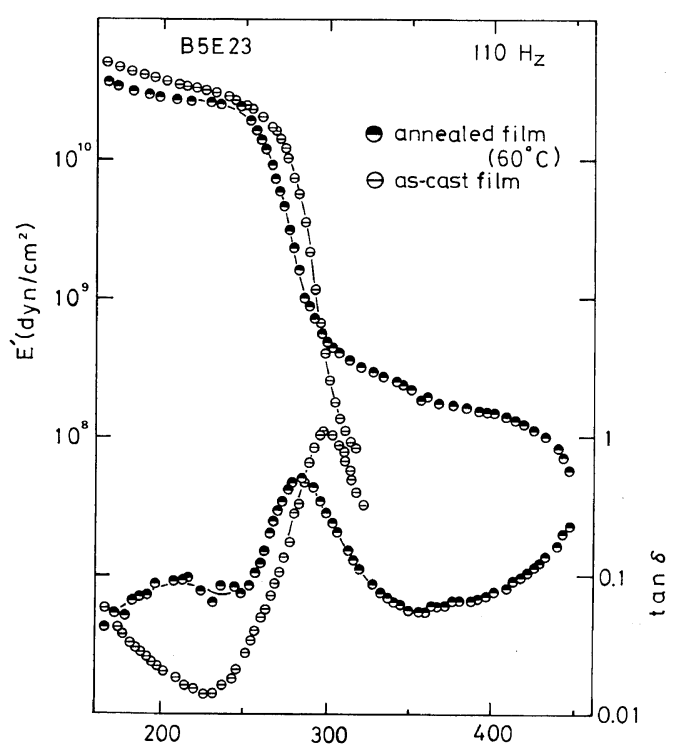

Figure 10. Effect of thermal treatment on temperature dependence of $E^{\prime}$ and $\tan \delta$ measured at $110 \mathrm{~Hz}$ on B5E23 sample. 
phase block copolymers. However, the DSC thermogram of the annealed B5E23 specimen was almost the same as that of the as-cast specimen. These results show that the annealing promoted microphase separation in the B5E23 specimen to some extent.

\section{DISCUSSION}

Crystallization and Melting of $P C$ and $P O E$ Crystallites. As was evidenced by DSC, dynamic mechanical measurements, and polarizing microscopic observation, the crystallization and melting of $\mathrm{PC}$ and POE segments are strongly influenced by the PC/POE composition and block length. The PC and POE crystallites coexist only at the PC/POE composition of $50 / 50$ by weight. When the PC content is large (PC content $\geqq 75 \%$ ), only PC crystallites exist, but with large POE content (PC content $\leqq 30 \%$ ), only POE crystallites exist. On consideration of the block length dependence of crystallization and melting behavior, it becomes evident that PC and POE segments are more easily crystallized with increasing block length. Table II, indicates that the degrees of crystallinity and the melting temperatures of both PC and POE crystallites become higher as a result of an increase in crystallite size with increasing block length.

It was shown in our previous paper ${ }^{7}$ that the crystallization rates of $\mathrm{PC}$ and POE blocks are influenced by adjoining segments. Thus, the crystallization rate of soft POE blocks was lessened by adjoining hard PC blocks, while that of hard PC blocks was accelerated by adjoining soft POE blocks. For B22E46 and B9E14 having large PC content, the specimens quenched from the melt usually showed no crystallinity, as is usually the case in PC homopolymers. ${ }^{18}$ Nevertheless, the quenched B13E68 specimen of 50/50 composition showed a PC melting peak preceded by PC-crystallization at $404 \mathrm{~K}$ during the reheating process. For B2E23 and B5E68 samples having large POE content the POEcrystallinity was smaller than that of POE homopolymers. For example, in an as-cast specimen of B5E23, the POE segments were not crystallized during the casting, but crystallization took place at about $270 \mathrm{~K}$ during the heating process. After being quenched from $540 \mathrm{~K}$ (2nd run), the B2E23 specimen showed single-phase random copolymer behavior.
In the following the spherulitic structure in $\mathrm{POE}$ rich B5E68 and B2E23 specimens will be considered. The spherulites became larger with increasing block length. The result is consistent with the results from DSC measurements. However, it is of interest that although DSC measurements on B5E68 showed only $50 \%$ crystallinity of POE segments, the Maltese crosses spread were spread over almost the entire specimen.

A similar spherulitic structure in multiblock copolymers was observed in segmented polyurethanes containing crystalline hard segments. A model of spherulitic superstructure was presented by Wilkes et al., ${ }^{19,20}$ who suggested that its overall morphology resembles fringed-micelles of semicrystalline polymers. However, this model differs greatly from the folded-chain model of spherulites. Furthermore, these authors suggested that the molecular axis of the hard crystalline segments preferentially orient in a radial, rather than tangential, direction, and the amorphous soft segments fill the space between the crystallites. However, the Wilkes model may not apply to our POE rich B5E68 and B2E23 specimens in which not hard PC segments but soft POE segments crystallize, according to the results of DSC measurements. In addition, stressstrain measurements showed that the B5E68 specimen exhibits a strain-induced plastic-to-rubber transition similar to that often found in thermoplastic elastomers. Thus, after yielding and necking, the B5E68 specimen could be extended to a $700 \%$ elongation (relative to the original length). The once-elongated specimen exhibited neither a POE

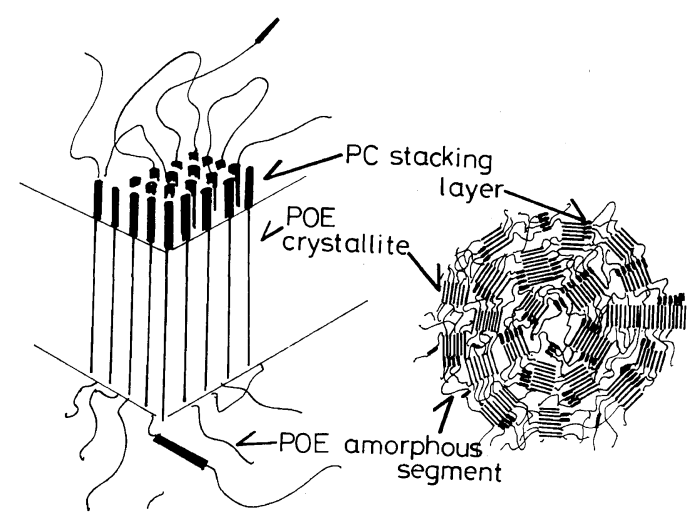

Figure 11. Schematic expression of a model for spherulitic structure of B5E68 and B2E23 samples. 
melting peak in DSC nor had a spherulitic structure under a polarizing microscope, but still possessed elastomeric properties allowing $300 \%$ recoverable elongation. These results suggest that the spherulitic structure and POE crystallites are easily disrupted by elongation, but that the PC hard segment domains, presumably incorporated in the spherulites, still act as physical crosslinks.

Figure 11 shows a morphology model of spherulitic structure by which our DSC data, polarizing microscopic observations, and stress-strain and dynamic mechanical data can be explained. This model has the following features: POE crystallites with PC stacking layers grow radially, while the molecular axis of POE crystallites aligns tangentially to the spherulite. POE amorphous segments fill the space between the crystallites. In the spherulite, one molecule passes through successive regions consisting of hard segment stacking layers, soft segment crystallites, and the amorphous regions. In the B5E68 specimen, the PC content is so low that PC crystallites cannot develop spontaneously. Probably, the crystalline POE regions facilitate the stacking of adjoining PC segments. This phenomenon may be called the "hard-segment stacking induced by soft-segment crystallization."

POE crystallites may be easily destroyed by stretching the specimen, thus undergoing yielding and necking. In such a specimen, the PC stacking regions would act as physical crosslinks, and thus impart to the specimen elastomeric properties. In fact, the B5E68 specimen exhibited a "plastic-torubber" transition similar to that found in SBS thermoplastic elastomers.

Acknowledgements. We should like to express our appreciation to Dr. T. Matsuda, Research Institute, National Cardiovascular Center, for his comments on the morphology of block copolymer spherulites. This work was supported in part by the Ministry of Education, Science and Culture (Mombusho) under Grants 147087 and 543026, which are gratefully acknowledged.

\section{REFERENCES}

1. M. Shen and H. Kawai, AIChE J., 24, 1 (1979).

2. T. Kotaka and M. Shen, Eds., Proceedings of U.S.-Japan Joint Seminar on Multicomponent Polymers, Kyoto, 1978; [J. Macromol. Sci., Phys., B17, No. 2-4 (1980)].

3. J. A. Manson and L. H. Sperling, "Polymer Blend and Composite," Plenum Press, New York, 1977, Chapter 5 .

4. J. W. C. Von Bogart, A. Lilaonitkal, and S. L. Cooper, Adv. Chem. Ser., 179, The American Chemical Society, Washington, D. C. (1979).

5. S. L. Cooper, J. Macromol. Sci., Phys., B17, 267 (1980).

6. T. Suzuki, K. Adachi, and T. Kotaka, Rep. Prog. Polym. Phys. Jpn., 21, 333 (1978).

7. T. Suzuki and T. Kotaka, Macromolecules, 13, 1495 (1980).

8. T. Suzuki, H. Chihara, and T. Kotaka, Kobunshigakkai Yokoshu (Polym. Prepr., Jpn.), 30, No. 9, 2170 (1981).

9. E. P. Goldberg, J. Polym. Sci., C, 4, 707 (1964).

10. T. Suzuki, MS Dissertation, Osaka, Univ., (1978).

11. K. Adachi, S. Hirose, and Y. Ishida, J. Polym. Sci., 13, 737 (1975).

12. T. G Fox and S. Loshaek, J. Polym. Sci., 15, 371 (1955).

13. J. A. Faucher, J. V. Koleske, E. R. Santee, J. J. Staratta, and C. W. Wilson, J. Appl. Phys., 37, 3962 (1966).

14. N. G. McCrum, B. E. Read, and G. Williams, "Anelastic and Dielectric Effects in Polymeric Solid," John Wiley, New York, 1976.

15. Y. Ishida, J. Polym. Sci., A-2, 7, 1835 (1969).

16. K. Se, K. Adachi, and T. Kotaka, Polym. J., 13, 1009 (1981).

17. M. L. Williams, R. F. Landel, and J. D. Ferry, J. Am. Chem. Soc., 77, 3701 (1955).

18. J. P. Mercier, J. J. Aklonis, M. Litt, and A. V. Tobolsky, J. Appl. Polym. Sci., 9, 447 (1965).

19. M. L. Samuel and G. L. Wilkes, Polym. Lett., 9, 761 (1971).

20. Y.-J. Chang and G. L. Wilkes, J. Polym. Sci., Polym. Phys. Eds., 13, 455 (1975). 